\title{
Coordination of timers and sensors in cell signaling
}

\author{
Junbin Qian ${ }^{1,2}$, Lendert Gelens ${ }^{3}$ and Mathieu Bollen ${ }^{1}$
}

${ }^{1}$ Laboratory of Biosignaling \& Therapeutics, KU Leuven Department of Cellular and Molecular Medicine, ${ }^{2}$ Laboratory of Translational Genetics, VIB Center for Cancer Biology, ${ }^{3}$ Laboratory of Dynamics in Biological Systems, KU Leuven Department of Cellular and Molecular Medicine, 3000 Leuven, Belgium

Correspondence: Junbin.Qian@kuleuven.vib.be; Lendert.Gelens@kuleuven.be; Mathieu.Bollen@kuleuven.be

Keywords: kinase, phosphatase, sensor, signaling integration, signaling profile, stress signaling, timer

Abbreviations: APC/C, anaphase promoting complex/cyclosome; KT-MT, kinetochoremicrotubule; MCC, mitotic checkpoint complex; NEB, nuclear envelope breakdown; SAC, spindle assembly checkpoint. 


\section{Abstract}

Timers and sensors are common devices that make our daily life safer, more convenient and more efficient. In a cellular context they arguably play an even more crucial role as they ensure the survival of cells in the presence of various extrinsic and intrinsic stresses. Biological timers and sensors generate distinct signaling profiles, enabling them to produce different types of cellular responses. Recent data suggest that they can work together to guarantee correct timing and responsiveness. By exploring examples of cellular stress signaling from mitosis, DNA damage and hypoxia, we discuss the common architecture of timer-sensor integration, and how its added features contribute to the generation of desired signaling profiles when dealing with stresses of variable duration and strength. We propose timer-sensor integration as a widespread mechanism with profound biological implications and therapeutic potential.

\section{Introduction}

Timers and sensors pervade our everyday life. Examples of simple timers include the typical kitchen timers and hourglasses. Many houses are also equipped with light and motion sensors (Fig. 1a). In engineering, timers and sensors are combined in a wide variety of ways to achieve certain functionalities. Whenever it becomes too dark outside, a light sensor detects this and turns on a lamp. However, as you may not want the light to remain on the whole night, a timer can be used to ensure that it is turned off a few hours after the sun sets. This illustrates a simple connection of one sensor and one timer (Fig. 1b, top), but additional functionalities can be introduced by combining multiple sensors and timers. For example, by adding a motion sensor and a second timer (Fig. 1b, bottom), the light can be made to go on automatically at night for a short while, whenever you go out. How one needs to connect different timers and sensors to design certain applications can be a challenging task, but it is one that engineers have mastered for a long time.

In biology, the importance of how cells sense their environment and adjust their behavior accordingly is widely recognized. Cells deal with various extrinsic or intrinsic stresses through signal transduction networks that are composed of sensor, transducer and effector proteins. ${ }^{[1]}$ An example of extrinsic stress is a shortage of oxygen (hypoxia), which results in an adaptive response that includes a transient cellcycle arrest and reduced energy consumption. However, progression through the cell 
cycle is also temporarily halted when chromosomes are damaged or improperly attached to microtubules during cell division, which are both examples of intrinsic stresses. Error-free cell-cycle progression is ensured by stress response mechanisms, also called checkpoints, which monitor the integrity of macromolecules or successful completion of a cell-cycle phase prior to initiating the next phase. Here, we propose that while sensors are a critical part of cellular responses to stress, they often operate in concert with timers to ensure an appropriate reaction. By exploring examples of stress signaling during mitosis, DNA damage and hypoxia, we find that the cellular response consists of a timer that operates in parallel with one or more sensors, arguing that this may be a common interaction motif in biology to ensure robust progression through the cell cycle and cell survival during stress conditions.

\section{Properties of biochemical timers and sensors}

The timers we use in our daily lives exist in multiple forms, such as digital or mechanical timers and typical hourglasses. The same is true for biological timers, which exploit macromolecules as building blocks. In all cases, however, a functional timer consists of two phases (Fig. 2a). In the first phase, just like winding up a clockwork or flipping the hourglass, an activating component ( $\mathrm{C}_{\text {act }}$ ) accumulates energy or activates effectors at a rate $r_{a c t .}$ Then, in a second phase, a deactivating component ( $\left.C_{\text {deact }}\right)$ is triggered with a certain time delay, which then leads to a release of the stored energy

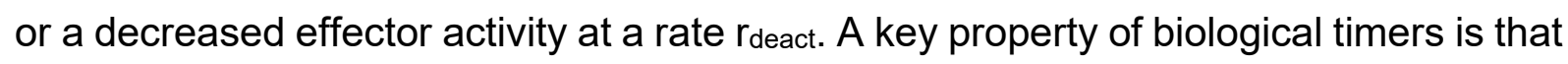
the output returns to its baseline values, despite the continued presence of the input signal, as long as the deactivating component remains active. ${ }^{[2-6]}$ Such a biphasic cellular response is embedded in signaling networks that promote the time-delayed activation of a deactivating component. Two well-characterized pulse-generating network circuits include incoherent feedforward loops and negative feedback loops (Fig. 2a). ${ }^{[2-4]}$ The incoherent feedforward loop is a circuit where the input has both a positive and negative effect on the output. This differs from a negative feedback loop in that the output now negatively regulates its own input. The exact duration of a biochemical timer depends on the (de)activation rates and the delay time involved, and is critically dependent on the underlying molecular mechanism. ${ }^{[4]}$ For example, a timer that is silenced by protein (de)phosphorylation is deactivated faster than a timer that is silenced by protein synthesis or degradation, simply because the latter processes often take more time. Similar network circuits have been studied in the context of adaptation, 
which is defined as a system that initially responds to a stimulus, but then returns to basal (perfect adaptation) or near-basal (near-perfect adaptation) levels after some transition period. ${ }^{[7,8]}$ Whereas the focus in adaptation lies on how good the basal levels are reached after this transition time, here we precisely emphasize this transition period as our 'timer'.

In many ways a sensor is simpler as it responds to the input with a response rate ract and $r_{\text {deact }}$ (Fig. 2b). The input lacks a deactivating component and merely relays or amplifies the input to the output. It remains active as long as the input is still there, and the effector is only inactivated upon withdrawal of the input stimuli. Because of this property, sensors are responsive to fluctuations of the input signal, regardless of timing.

Timers and sensors thus have their own advantages and disadvantages, which make them suitable for different biological circumstances. However, it seems to be a recurrent theme that timers and sensors work together to ensure correct timing and responsiveness in cellular stress signaling. It is well established that cell-cycle progression depends on a number of checkpoints that evaluate whether the cell can proceed to the next cell-cycle phase (Fig. 2c). Whether a checkpoint is activated and halts cell-cycle progression depends on the level of stress that is detected by appropriate sensors. A cell-cycle arrest can be transient until the underlying problem is solved, e.g. through proper attachment of chromosomes to the microtubule spindle, DNA repair, or an increased supply of oxygen. However, if the cause of the stress cannot be solved the cell-cycle arrest can become permanent and eventually culminate in senescence or cell death. As we will illustrate in the next sections, in all of these cases, a timer is integrated with one or more sensors. While the sensor continuously integrates the (possibly fluctuating) cellular stress levels, the timer can be activated by either cellular stress or entering a new cell-cycle phase. By starting a timer that transiently activates a stress response, it provides the cell with a time window in which it can integrate the stress signals and decide whether or not to activate a checkpoint or adaptive response (Fig. 2d).

\section{Timer-sensor coordination ensures robust cellular responses}

\subsection{The spindle-assembly checkpoint}


The spindle-assembly checkpoint (SAC) or mitotic checkpoint is a mitotic surveillance mechanism that delays the onset of anaphase until all chromosomes are properly attached to spindle microtubules (MT) via protein complexes, known as kinetochores $(\mathrm{KT})$, that are assembled on centromeric DNA. ${ }^{[9]} \mathrm{SAC}$ signaling generates a multimeric mitotic checkpoint complex (MCC), which prevents activation of the anaphasepromoting complex/cyclosome (APC/C). The APC/C is an E3-type ubiquitin ligase that initiates chromosome segregation during anaphase. MCC assembly is scaffolded by a key SAC protein, MAD1, which is recruited to the kinetochores after nuclear envelope breakdown (NEB) (Table 1). ${ }^{[10]}$ MAD1 recruitment is not only mediated by protein kinase BUB1, but also by two other kinetochore receptors, namely the RZZ (RODZwilch-ZW10) complex and CEP57.[11-14] Because MAD1 is only recruited to unattached kinetochores, while KT-MT interactions result in the removal of MAD1 from kinetochores, it is generally accepted that MAD1 recruitment is regulated by attachment sensors. ${ }^{[15]}$ Indeed, attachment is responsible for the removal of the kinetochore MAD1 pools of the RZZ and CEP57 pathways. ${ }^{[11,16,17]}$ However, we recently found that the BUB1 pathway for MAD1 recruitment operates independently of attachment. ${ }^{[18]}$ Mechanistically, unattached kinetochores recruit the SAC-activating kinase MPS1, which promotes the kinetochore recruitment of BUB1 (Fig. 3a). In addition, MPS1 promotes the BUB1:MAD1 interaction by phosphorylating BUB1 at Thr461. ${ }^{[18-20]}$ Furthermore, MPS1 induces kinetochore recruitment of BUBR1, which then recruits the deactivating component, protein phosphatase PP2A-B56, to dephosphorylate BUB1 at Thr461. ${ }^{[18]}$ However, the recruitment of PP2A-B56 via BUBR1 only occurs after a delay time. Indeed, while BUB1-Thr461 phosphorylation by MPS1 already starts in early prophase, BUBR1 is only recruited to the kinetochores after NEB. Moreover, the PP2A-B56 binding capacity of BUBR1 requires its prior phosphorylation at the kinetochores by the mitotic kinases CDK1 and PLK1. Thus, MPS1 not only directly phosphorylates BUB1-Thr461, but also indirectly promotes BUB1-Thr461 dephosphorylation after a time delay. This incoherent feedforward loop generates a biphasic response of MAD1 recruitment and corresponding MCC assembly. ${ }^{[18]}$ More importantly, this BUB1-centered biochemical timer ensures that the BUB1:MAD1 interaction is attachment-independent, as BUB1-Thr461 dephosphorylation occurs after a defined time, even in the presence of completely unattached kinetochores. 


\subsection{Microtubule-kinetochore attachments}

In early mitosis MT-KT attachments are continuously monitored to ensure that the duplicated chromosomes become bi-oriented, meaning that the sister chromatids are connected to MTs from opposing poles. ${ }^{[21]}$ Bi-orientation generates inter- and intrakinetochore tension. As long as the chromosomes are not bi-oriented, e.g. because only one sister chromatid is connected to the spindle or because they are both connected to the same spindle pole, there is no tension and the KT-MT interface is in close physical proximity to the centromeric pool of protein kinase Aurora B. This enables the phosphorylation of key regulatory proteins at the kinetochores by Aurora $B$ and results in the detachment of associated MTs. This Aurora-B-dependent tensionsensing mechanism of KT-MT interaction monitoring applies to each individual pair of sister chromatids.

Compelling data suggest that MT-KT attachments are also regulated at a global level and are generally unstable during prometaphase, even at bi-oriented chromosomes. ${ }^{[22]}$ At the prometaphase-metaphase transition, chromosomes collectively undergo a coordinated switch from relatively unstable to stable MT-KT attachments. This collective behavior is explained by a Cyclin-A degradation timer, which starts in early prometaphase and is completed by metaphase. Cyclin A activates CDK1 in early mitosis, which then activates the deactivating component $A P C / C$ to target Cyclin A for proteasomal degradation (Fig. $3 b$ ). Since Cyclin A/CDK1 destabilizes MT-KT attachments, the progressive degradation of Cyclin A results in a gradual and global stabilization of MT-KT attachments. ${ }^{[22,23]}$ The degradation of Cyclin A is embedded in a negative feedback loop between Cyclin A/CDK1 and APC/C, and co-regulates MT$\mathrm{KT}$ attachments with centromeric Aurora B. However, in contrast to the Aurora-B controlled sensor, the Cyclin-A degradation timer is irreversible and targets all chromosomes simultaneously. The collaboration between the centromeric Aurora- $B$ sensor and the cytoplasmic Cyclin-A degradation timer ensures a coordinated autonomous and collective response of MT-KT dynamics, needed for faithful chromosome segregation.

\subsection{The DNA-damage response during the $\mathbf{G} 2$ checkpoint}

DNA double-strand breaks (DSBs) in the $G 2$ phase of the cell cycle activate the $G 2$ checkpoint and result in the activation of the DNA-damage-response (DDR) pathway, 
which senses and repairs DSBs. ${ }^{[24]}$ The DDR is associated with a G2 cell-cycle arrest providing the time needed for DNA repair and for preventing aneuploidy (abnormal number of chromosomes) and the propagation of mutations to progeny. If the DDR sensor were the only component of the G2 checkpoint, cell-cycle progression would resume only after the repair of all DNA lesions. However, accumulating evidence suggests that cells can bypass the $G 2$ checkpoint, even in the presence of a few unrepaired DNA lesions, in both cancer and untransformed cells. ${ }^{[25-28]}$ The activating components of the G2 checkpoint are protein kinases ATM (Ataxia Telangiectasia Mutated) and ATR (Ataxia Telangiectasia and Rad3 related), which phosphorylate and activate multiple cell-cycle arrest and DNA repair effectors (Table 1). ${ }^{[28]}$ Despite having a similar substrate specificity, ATM and ATR operate with a distinct time frame. ATM responds faster to DNA break signals than does ATR, and the dephosphorylation of ATM substrates also precedes that of ATR substrates, even when ATM remains active and some DSBs still persist. ${ }^{[28,29]}$ The dephosphorylation of ATM substrates is associated with a resumption of cell-cycle progression and is mediated by a chromatinbound deactivating component, i.e. protein phosphatase PPM1D/WIP1 (Wild-type p53induced phosphatase 1). Intriguingly, the amount of WIP1 is increased by ATM in at least two ways. First, ATM phosphorylates and stabilizes p53, which promotes the transcription of WIP1. ${ }^{[30]}$ Second, ATM activates the AMP-activated protein kinase (AMPK), which phosphorylates HIPK2 (Homeodomain-Interacting Protein Kinase 2) to disrupt its interaction with WIP1. Since WIP1 is targeted for proteasomal degradation following its phosphorylation by HIPK2, the dissociation of HIPK2 stabilizes WIP1. ${ }^{[31]}$ This double incoherent feedforward regulation explains why the ATM response is transient (Fig. 3c). On the one hand, the interplay between ATM and WIP1 generates a pulse response that sets the minimal duration for recovery from the $G 2$ checkpoint. On the other hand, ATR activation occurs later and its substrates remain phosphorylated as long as DNA damage persists, suggesting that the ATR pathway operates as a genuine sensor. ${ }^{[28]}$ Interestingly, the ATM timer and ATR sensor are functionally connected as ATM-dependent DNA-end resection provides the single strand DNA ends needed for ATR recruitment and activation. ${ }^{[32]}$ Such a timer-sensor connection was recently proposed to ensure an appropriate response to distinct levels of DNA damage, i.e. a delayed mitotic entry when there are only few unrepaired DNA damage sites and no mitotic entry, or even senescence or cell death, when the DNA damage is excessive. ${ }^{[28,33]}$ 


\subsection{The hypoxic stress response}

Timer-sensor integration also explains how cells cope with a decreased oxygen supply, which induces the hypoxic stress response. This adaptive response is primarily executed by two hypoxia-inducible factors (HIF), HIF-1 $\alpha$ and HIF-2 $\alpha$. Under normoxic conditions, HIF-1/2 $\alpha$ are rapidly hydroxylated by specific prolyl hydroxylases (PHDs) in an oxygen-dependent manner. ${ }^{[34-36]}$ The hydroxylation of HIF-1/2 $\alpha$ enables their ubiquitination by the E3 ubiquitin ligase von Hippel-Lindau ( $p V H L)$ and targets them for proteasomal degradation. Under hypoxic conditions, HIF-1/2 $\alpha$ are stabilized due to inhibition of their PHDs. This allows the nuclear entry of HIF- $1 \alpha$ and HIF- $2 \alpha$, and their heterodimerization with HIF-1 $\beta$. These heterodimeric complexes bind to the hypoxia responsive element (HRE), an enhancer that is associated with hypoxia-responsive genes, and causes the transactivation of these genes (Fig. 3d). However, HIF-1 $\alpha$ and HIF-2 $\alpha$ are not completely redundant. Indeed, HIF-1 $\alpha$ is stabilized earlier (2-24h) and therefore acts more quickly to acute hypoxia. ${ }^{[35]}$ On the other hand, the activity of HIF$1 \alpha$ is more transient as the protein is gradually degraded during prolonged hypoxia (24-48h). Therefore, despite its slower stabilization kinetics, HIF-2 $\alpha$ ultimately surpasses the level of HIF-1 $\alpha$ during prolonged hypoxia ( $>24 \mathrm{~h}$ ) and dominates the regulation of chronic hypoxia, which is referred to as the HIF switch. ${ }^{[35]}$ The deactivation phase of HIF-1 $\alpha$ is explained by its coupling to two negative feedback loops (Table 1 and Fig. 3d). First, HIF-1 $\alpha$ activation leads to the expression of a deactivating component, HIF1A antisense RNA, which binds to the HIF-1 $\alpha$ transcript and targets it for degradation. ${ }^{[37]}$ Second, HIF-1 $\alpha$ induces the expression of another deactivating component, HSP70, that binds to the E3 ubiquitin ligase CHIP and selectively targets the HIF-1 $\alpha$ protein for degradation. ${ }^{[38,39]}$ In addition, prolonged hypoxia leads to the expression of Hypoxia-Associated Factor (HAF), which selectively binds and degrades HIF- $1 \alpha$ in an oxygen-independent manner but promotes HIF-2 $\alpha$ transactivation and stabilization. ${ }^{[40]}$ This HIF-1/2 $\alpha$ signaling network structure generates a pulse-like HIF$1 \alpha$ response and a slower but sustained HIF-2 $\alpha$ response (Fig. $3 d$ and $3 e$ ). Hence, the coordination of the HIF-1 $\alpha$ timer and HIF-2 $\alpha$ sensor enables cells to generate an appropriate adaptive response to both acute and chronic hypoxia. 


\section{Biological implications of timer-sensor coordination}

Based on the examples presented above, we suggest that timer-sensor integration is needed to generate an appropriate cellular response to various stress signals. First, since a timer often responds faster than an associated sensor, it generates a time window in which an adaptive response is transiently activated as a safety measure. During this time, the sensor can evaluate whether the input (e.g. an extrinsic or intrinsic stress signal) needs a response for a more extended time (e.g. by checkpoint activation). If the timer is too short, there does exist a risk that critical stress signals are insufficient to elicit a proper response. Second, since sensor-induced signaling does not need to be fully operational right away and may even never have to be turned on if a complete and successful response is already elicited during the time provided by the timer, timer-sensor coordination may represent a means to conserve energy. This may be particularly true for fluctuating input signals which require continuous, energy consuming sensor on-off signaling (SAC, hypoxic response). Third, chronic sensor activation can lead to irreversible cell fate decisions such as senescence or cell death. When the sensor is connected with a timer, irreversible cell fate decisions can be delayed until the timer is silenced (DNA-damage response, hypoxic response). Finally, when the timer and sensor are triggered at different locations, they can coordinate more complex activities, such as a spatially coordinated response (MT-KT attachments).

\section{Therapeutic implications}

Drugs that interfere with cell-cycle progression, including DNA-damaging agents and anti-mitotic drugs, are clinically used for cancer therapy. ${ }^{[41]}$ These therapies are aimed at preventing cancer-cell proliferation by chronically activating cell-cycle checkpoints. Such therapies mainly target sensor pathways and often lead to drug resistance rather quickly. We hypothesize that the chronic activation of both timer and sensor pathways is not only a more efficient therapy but is also less likely to elicit drug resistance because it inhibits at least two distinct targets. To inhibit the silencing of biochemical timers we propose to target the key deactivating component in the timer network circuit. For example, the recruitment of PP2A-B56 drives the termination of the BUB1 timer; abolishing PP2A-B56 recruitment by mutation of its binding site on BUBR1 not only effectively prevented the deactivation of the BUB1 timer, but also kept cancer cells 
arrested in mitosis for a sufficient long time to provoke cell death. ${ }^{[18]}$ Similarly, targeting the WIP1 phosphatase chronically activates the ATM timer during the DNA-damage response. Indeed, genetic ablation or chemical inhibition of WIP1 has been reported to kill cancer cells, especially when combined with a DNA-damaging reagent. ${ }^{[42,43]}$

Hypoxia has also been studied extensively as a target for cancer therapy. ${ }^{[44]}$ However, its clinical benefit is context dependent, which can be explained by the overlapping but non-identical functions of HIF-1 $\alpha$ and HIF-2 $\alpha .{ }^{[35,45]}$ While there is a consensus on the role of HIF-2 $\alpha$ as an oncoprotein, the function of HIF-1 $\alpha$ appears to be more pleiotropic. ${ }^{[35,36]}$ Indeed, HIF-1 $\alpha$ promotes initial tumor growth in hypoxia but limits cancer proliferation when it acts chronically. ${ }^{[46]}$ Hence, a novel cancer treatment strategy could be to simultaneously activate HIF-1 $\alpha$ and inhibit HIF-2 $\alpha$. If HIF-1 $\alpha$ signaling indeed functions as a timer, the inhibition of its deactivating components may kill cancer cells during chronic hypoxia. Consistent with this notion, targeting the antisense transcripts of HIF-1 $\alpha$ (AS-HIF1A) has been shown to suppress cancer cell proliferation (Fig. 3d). ${ }^{[47]}$ Another attractive target in this respect is $\mathrm{HAF}$, because its downregulation stabilizes HIF-1 $\alpha$ but inactivates HIF-2 $\alpha .{ }^{[40]}$ In contrast, pVHL does not appear to be a suitable target as its inhibition leads to stabilization of both HIF-1 $\alpha$ and HIF-2 $\alpha$.

\section{Conclusions and prospects}

Pathway redundancy is a common choice of evolution to provide a back-up system in case the canonical pathway fails to respond. However, timer-sensor integration is not a simple back-up system, as it provides additional advantages for cells to generate adaptive responses, especially in multicellular organisms that are faced with a broad variety of environmental challenges. We propose that timer-sensor coordination is widespread in biological processes and may even have more benefits than currently appreciated. It will be crucial to further identify and mechanistically dissect the key components of timer-sensor integration, as this may lead to a better understanding of cellular responses in both physiological and pathological conditions. Particular attention should be given to the crosstalk between timers and sensors, because it is probably the key to generate desired signaling profiles and its failure may cause diseases. We propose that the combined targeting of the timer and sensor can be strategically used to generate a more efficient patient response and prevent drug 
resistance. Finally, for the specific examples discussed in this review, the timer duration varies from $\sim 30$ min to $\sim 48$ hours due to differences in the mechanisms underlying the recruitment and/or activation of the deactivating component. Having a particular duration of a timer may have an evolutionary advantage in that it is optimized for multiple parameters, i.e. robustness, speed, and energy consumption. Just as engineers use a multitude of combinations of timers and sensors to realize a wide variety of applications, evolution seems to have found its own ways to coordinate complex tasks by optimally combining timers and sensors. Understanding these biological optimization strategies may even inspire engineers to develop new types of timer-sensor based equipment.

\section{ACKNOWLEDGEMENTS}

This work was supported by the Fund for Scientific Research-Flanders (grant G0B9917N and GOA5317N), the KU Leuven Research Fund (GOA/15/016 and C14/18/084), and the Belgian Foundation Against Cancer.

\section{REFERENCES}

[1] D. Kültz, Annu. Rev. Physiol. 2005, 67, 225.

[2] Y. Hart, U. Alon, Mol. Cell 2013, 49, 213.

[3] S. Mangan, U. Alon, Proc. Natl. Acad. Sci. 2003, 100, 11980.

[4] L. Gelens, J. Qian, M. Bollen, A. T. Saurin, Trends Cell Biol. 2018, 28, 6.

[5] J. J. Tyson, K. C. Chen, B. Novak, Curr. Opin. Cell Biol. 2003, DOI 10.1016/S0955-0674(03)00017-6.

[6] J. J. Tyson, B. Novák, Annu. Rev. Phys. Chem. 2010, DOI 10.1146/annurev. physchem.012809.103457.

[7] W. Ma, A. Trusina, H. El-Samad, W. A. Lim, C. Tang, Cell 2009, 138, 760.

[8] J. E. Ferrell, Cell Syst. 2016, 2, 62.

[9] A. Musacchio, Curr. Biol. 2015, 25, R1002.

[10] Y. Luo, E. Ahmad, S.-T. Liu, Front. Cell Dev. Biol. 2018, 6, 1. 
[11] H. Zhou, T. Wang, T. Zheng, J. Teng, J. Chen, Nat. Commun. 2016, 7, 10151.

[12] G. J. P. L. Kops, Y. Kim, B. A. A. Weaver, Y. Mao, I. McLeod, J. R. Yates, M. Tagaya, D. W. Cleveland, J. Cell Biol. 2005, 169, 49.

[13] L. Défachelles, N. Raich, R. Terracol, X. Baudin, B. Williams, M. Goldberg, R. E. Karess, Chromosome Res. 2015, 23, 333.

[14] G. Zhang, T. Lischetti, D. G. Hayward, J. Nilsson, Nat. Commun. 2015, 6, 7162.

[15] B. Etemad, G. J. Kops, Curr. Opin. Cell Biol. 2016, 39, 101.

[16] and T. S. H. Sarah Mische, Yungui He, Lingzhi Ma, Mingang Li, Madeline Serr, Mol. Biol. Cell 2008, DOI 10.1091/mbc.E08.

[17] M. V. S. Sivaram, T. L. Wadzinski, S. D. Redick, T. Manna, S. J. Doxsey, EMBO J. 2009, DOI 10.1038/emboj.2009.38.

[18] J. Qian, M. A. García-Gimeno, M. Beullens, M. G. Manzione, G. Van der Hoeven, J. C. Igual, M. Heredia, P. Sanz, L. Gelens, M. Bollen, Mol. Cell 2017, 68, 715.

[19] G. Zhang, T. Kruse, B. López-Méndez, K. B. Sylvestersen, D. H. Garvanska, S. Schopper, M. L. Nielsen, J. Nilsson, Nat. Commun. 2017, 8, 15822.

[20] Z. Ji, H. Gao, L. Jia, B. Li, H. Yu, Elife 2017, 6, 1.

[21] K. M. Godek, L. Kabeche, D. A. Compton, Nat. Rev. Mol. Cell Biol. 2015, 16, 57.

[22] L. Kabeche, D. a. Compton, Nature 2013, DOI 10.1038/nature12507.

[23] A. M. G. Dumitru, S. F. Rusin, A. E. M. Clark, A. N. Kettenbach, D. A. Compton, Elife 2017, 6, 1.

[24] I. A. Shaltiel, L. Krenning, W. Bruinsma, R. H. Medema, J. Cell Sci. 2015, 128, 607.

[25] D. Deckbar, J. Birraux, A. Krempler, L. Tchouandong, A. Beucher, S. Walker, T. Stiff, P. Jeggo, M. Löbrich, J. Cell Biol. 2007, DOI 10.1083/jcb.200612047.

[26] K. Tkacz-Stachowska, C. Lund-Andersen, A. Velissarou, J. H. Myklebust, T. Stokke, R. G. Syljuåsen, Radiother. Oncol. 2011, DOI 10.1016/j.radonc.2011.05.060. 
[27] A. Loewer, K. Karanam, C. Mock, G. Lahav, BMC Biol. 2013, DOI 10.1186/17417007-11-114.

[28] H. Jaiswal, J. Benada, E. Müllers, K. Akopyan, K. Burdova, T. Koolmeister, T. Helleday, R. H. Medema, L. Macurek, A. Lindqvist, EMBO J. 2017, 36, 2161.

[29] J. van den Berg, A. G. Manjón, K. Kielbassa, F. M. Feringa, R. Freire, R. H. Medema, Nucleic Acids Res. 2018, 1.

[30] S. Shreeram, O. N. Demidov, W. K. Hee, H. Yamaguchi, N. Onishi, C. Kek, O. N. Timofeev, C. Dudgeon, A. J. Fornace, C. W. Anderson, Y. Minami, E. Appella, D. V. Bulavin, Mol. Cell 2006, DOI 10.1016/j.molcel.2006.07.010.

[31] D. W. Choi, W. Na, M. H. Kabir, E. Yi, S. Kwon, J. Yeom, J. W. Ahn, H. H. Choi, Y. Lee, K. W. Seo, M. K. Shin, S. H. Park, H. Y. Yoo, K. ichi Isono, H. Koseki, S. T. Kim, C. Lee, Y. K. Kwon, C. Y. Choi, Mol. Cell 2013, 51, 374.

[32] A. Jazayeri, J. Falck, C. Lukas, J. Bartek, G. C. M. Smith, J. Lukas, S. P. Jackson, Nat. Cell Biol. 2006, DOI 10.1038/ncb1337.

[33] F. M. Feringa, J. A. Raaijmakers, M. A. Hadders, C. Vaarting, L. Macurek, L. Heitink, L. Krenning, R. H. Medema, Nat. Commun. 2018, 9, 3923.

[34] H. Choudhry, A. L. Harris, Cell Metab. 2017, DOI 10.1016/j.cmet.2017.10.005.

[35] M. Y. Koh, G. Powis, Trends Biochem. Sci. 2012, 37, 364.

[36] B. Keith, R. S. Johnson, M. C. Simon, Nat. Rev. Cancer 2011, 12, 9.

[37] F. Rossignol, C. Vaché, E. Clottes, Gene 2002, 299, 135.

[38] W. Luo, J. Zhong, R. Chang, H. Hu, A. Pandey, G. L. Semenza, J. Biol. Chem. 2010, DOI 10.1074/jbc.M109.068577.

[39] S. Tsuchida, Y. Arai, K. A. Takahashi, T. Kishida, R. Terauchi, K. Honjo, S. Nakagawa, H. Inoue, K. Ikoma, K. Ueshima, T. Matsuki, O. Mazda, T. Kubo, J. Orthop. Res. 2014, 32, 975.

[40] M. Y. Koh, R. Lemos, X. Liu, G. Powis, Cancer Res. 2011, DOI 10.1158/00085472.CAN-10-4142.

[41] T. Otto, P. Sicinski, Nat. Rev. Cancer 2017, 17, 93. 
[42] W. Kong, X. Jiang, W. E. Mercer, Cancer Biol. Ther. 2009, 8, 555.

[43] S. Rayter, R. Elliott, J. Travers, M. G. Rowlands, T. B. Richardson, K. Boxall, K. Jones, S. Linardopoulos, P. Workman, W. Aherne, C. J. Lord, A. Ashworth, Oncogene 2008, 27, 1036.

[44] W. R. Wilson, M. P. Hay, Nat. Rev. Cancer 2011, DOI 10.1038/nrc3064.

[45] G. L. Semenza, Trends Pharmacol. Sci. 2012, DOI 10.1016/j.tips.2012.01.005.

[46] C. Shen, R. Beroukhim, S. E. Schumacher, J. Zhou, M. Chang, S. Signoretti, W. G. Kaelin, Cancer Discov. 2011, 1, 222.

[47] M. Chen, C. Zhuang, Y. Liu, J. Li, F. Dai, M. Xia, Y. Zhan, J. Lin, Z. Chen, A. He, W. Xu, G. Zhao, Y. Guo, Z. Cai, W. Huang, Cancer Lett. 2016, 376, 155. 


\section{LEGENDS TO THE FIGURES}

Figure 1. Timer-sensor integration in daily life. a) Sketch of the response of a timer and a sensor to similar inputs. b) Wiring diagram of how a light sensor, motion sensor, and different timers can be integrated to control whether a lamp is turned on or off.

Figure 2. Differences of biological timers and sensors. a) Basic signaling networks that elicit a biphasic response. Such a timer involves two time scales: the rate of activation $\left(r_{\text {act }}\right)$ and the rate of deactivation $\left(r_{\text {deact }}\right)$. b) A sensor also has such response rates but, in contrast to the timer, it only deactivates after removal of the input. c) The cell-cycle regulatory network consists of many checkpoints and stress response systems, which could all involve a combination of timers and sensors. d) Example of how a timer and sensor can work together to achieve a desired response.

Figure 3. Network signaling diagram of timers and sensors during SAC signaling (a), kinetochore-microtubule (MT-KT) attachment in prometaphase (b), DNA damage in $\mathrm{G} 2$ (c), and hypoxia (d,e). 


\section{a Timer}

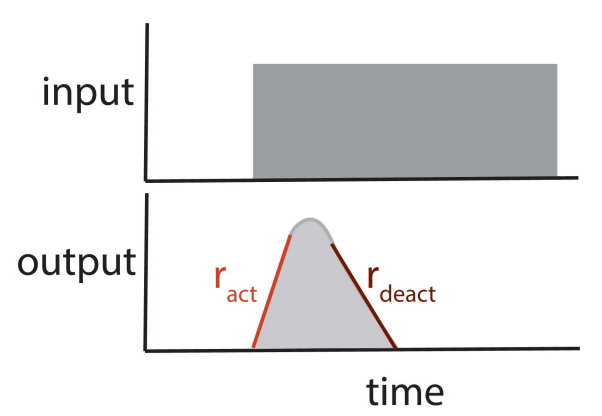

C

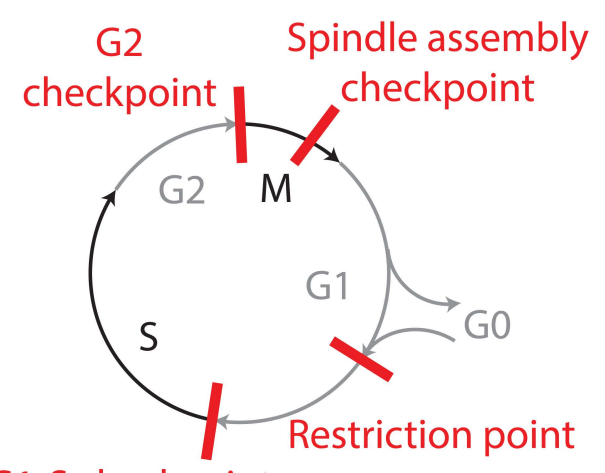

G1-S checkpoint

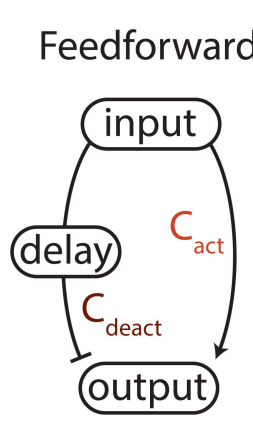

b Sensor

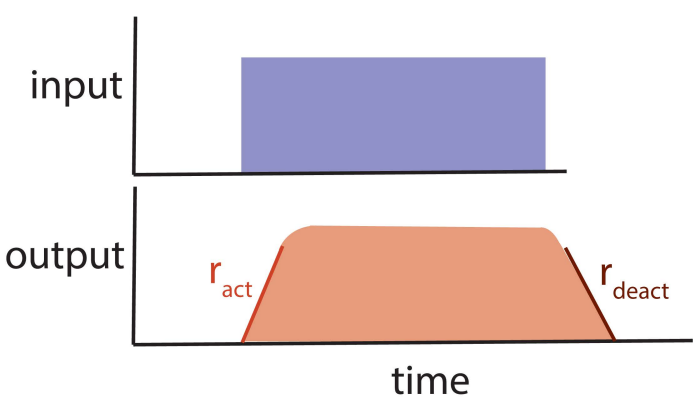

d

\section{Intrinsic stress}

- DNA damage

- DNA replication

- Incorrect spindle assembly

- Small cell size

\section{Extrinsic stress}

- Lack of growth factors

- Lack of nutrients

- Hypoxia start timer

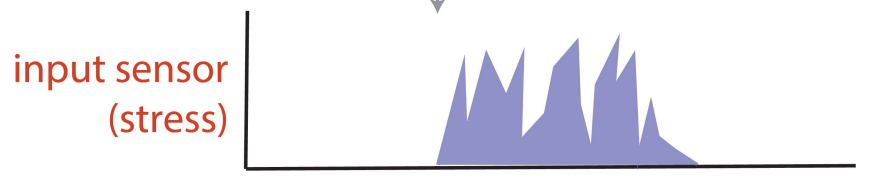

output

time 


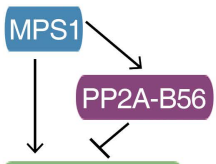

BUB1-T461ph

$\downarrow$

MCC

BUB1 timer

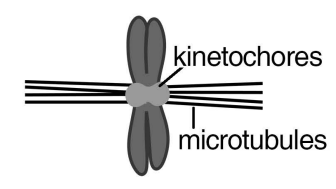

Attached

Unattached

$y$

MCC

Attachment sensor

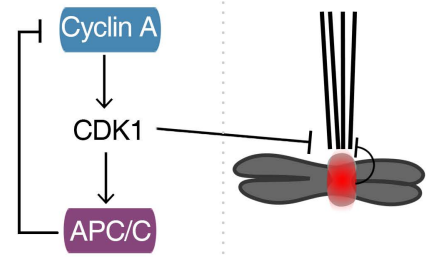

Cytoplasm:

Cyclin A timer
Centromere: Aurora B sensor
C

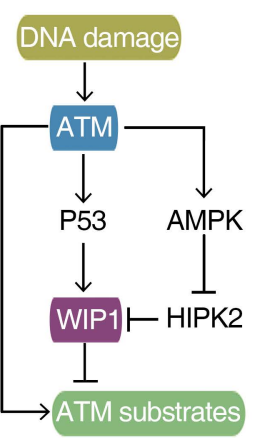

d

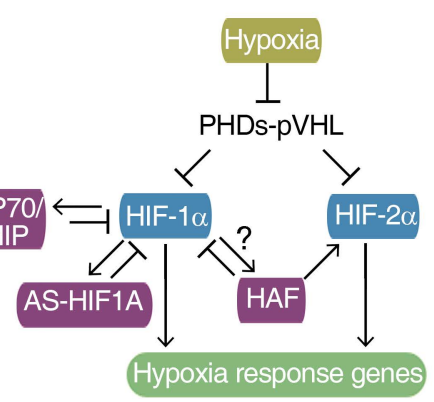

e

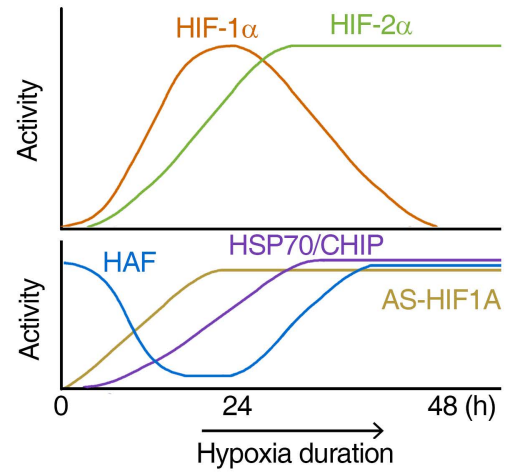


Table 1. Examples of timer-sensor integration

\begin{tabular}{llllll}
\hline $\begin{array}{l}\text { Cellular } \\
\text { process }\end{array}$ & $\begin{array}{l}\text { Shared } \\
\text { target }\end{array}$ & Timer & $\begin{array}{l}\text { Activating } \\
\text { component }\end{array}$ & $\begin{array}{l}\text { Deactivating } \\
\text { component }\end{array}$ & Sensor \\
\hline SAC & MAD1 & BUB1 & MPS1 & PP2A-B56 & CEP57/RZZ \\
\hline $\begin{array}{l}\text { MT-KT } \\
\text { attachment }\end{array}$ & NDC80 & Cyclin A & CDK1 & APC/C & Aurora B \\
\hline G2 checkpoint & $\begin{array}{l}\gamma \mathrm{H} 2 \text { AX/p53 } \\
\text { CDC25/PLK1 }\end{array}$ & ATM & ATM & WIP1 & ATR \\
\hline $\begin{array}{l}\text { Hypoxia } \\
\text { response }\end{array}$ & $\begin{array}{l}\text { Hypoxia } \\
\text { responsive } \\
\text { genes }\end{array}$ & HIF-1 $\alpha$ & HIF-1 $\alpha$ & $\begin{array}{l}\text { HAF/AS- } \\
\text { HIF1A/HSP70- } \\
\text { CHIP }\end{array}$ & HIF-2 $\alpha$ \\
\hline
\end{tabular}

